\title{
Calculation of Mutual Inductance Based on 3D Field and Circuit Coupling Analysis for WPT System
}

\author{
Yang $\operatorname{Han}^{\mathrm{a}}$ and Xiaoming Wang ${ }^{\mathrm{b}}$ \\ Department of Electrical Engineering and Automation, Harbin Institute of \\ Technology, Harbin \\ a.hanyang111@126.com,b.xmw2002@163.com
}

\begin{abstract}
Determination of mutual inductance in the WPT system based on the electromagnetic coupling mode is crucial for system design and efficiency optimization. The analytic formulas and experimental methods which only aim at a coupling coil with a certain type of configuration and parameters are not universal approaches. Therefore they can't serve as a design basis for the system optimization. This paper presents a novel method based on $3 D$ field and circuit coupling analysis to calculate mutual inductance of coupling coils with arbitrary spatial distribution for WPT system. The method can obtain maximum efficiency under the condition that the spatial distribution of coupling coils are irregular and restrained in actual physical systems. The method has made a breakthrough and provided a powerful tool for the design and optimization of electromagnetic coupling power transmission system.
\end{abstract}

Keywords: Mutual inductance, field-circuit coupling, FEA, WPT

\section{Introduction}

As a fundamental electrical parameter, mutual inductance's practical usage appears in numerous fields and applications, such as all ranges of transformers [6], applications of wireless power transmission [1,2,3,7,8,14], generators, motors, magnetic resonance applications [5,9], medical electronic devices [10], etc. Many of the mentioned areas require precise computational results of mutual inductance. Take WPT applications for example, numerous WPT systems use the configuration of two electromagnetic coupling coaxial circular coils with rectangular cross section. Many factors affect the coupling efficiency such as axial distance, lateral deviation, angular deviation, etc. There are numerous approaches and techniques for calculating mutual inductance, by using elliptic integrals of the first and second kind of Heuman's lambda function [11,12], Neumann functions [4,11], Bessel functions, or a combination of Bessel, Struve and Legendre functions [13], etc. However, it is difficult to propose a practical formula through analytical methods on the condition of irregular geometrical shape and particular spatial position of coils.

The paper proposes a navel approach based on 3D field and circuit coupling analysis for calculating mutual inductance of power transmission system with electromagnetic coupling coils and utilizes the results to obtain system transmission efficiency. The new method models, analyses and calculates the coupling coils, then the configuration and spatial distribution of the coupling coils can be intuitively analyzed and improved from the rate of the secondary coil receiving magnetic field. The secondary open circuit voltage and the primary current with short circuit for the secondary are obtained by calculating and analyzing based on field-circuit coupling, and then the primary self-inductance and the mutual inductance of coils are also obtained. The variety law of the mutual inductance and the power transmission efficiency of the two electromagnetic coupling coils can be calculated by changing the coupling coils spatial distribution parameters.[15] 


\section{Hollow Solenoid Coil Model and Finite Simulation}

Common spatial distribution of the coils are arranged in coaxial in the topology of power transmission system with two coupling coils, however, the paper gives some other cases uncommon, for example lateral deviation, angular deviation and embedded position. The paper establishes a model in order to simplify the analysis, and the parameters of the two hollow solenoid coils are shown in Figure 1.

For the two hollow solenoid coils, the axial distance between the coils is d; the turns, the inner radius, and the outer radius of the two coils are respectively $N, R_{1}$, $R_{3}, \quad R_{2}, R_{4}$; the height and the cross section area of the coils are respectively $\mathrm{h}$, $2\left(R_{2}-R_{1}\right) \times h$, and $2\left(R_{4}-R_{3}\right) \times h$.

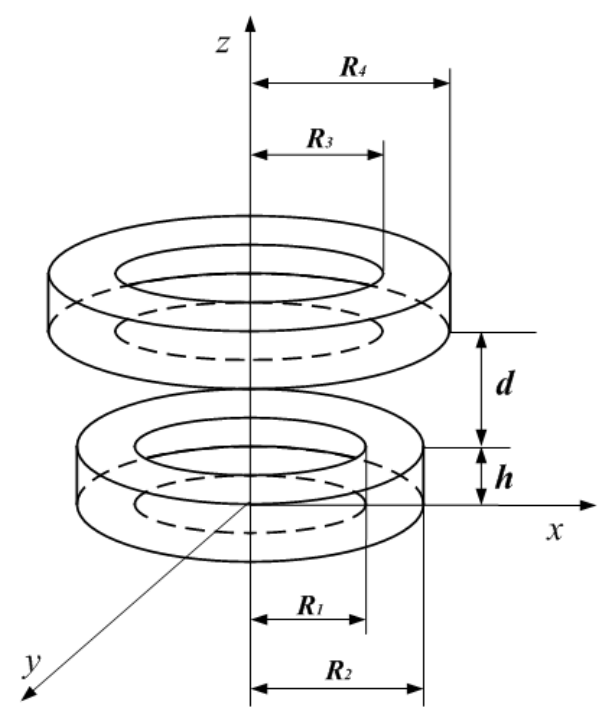

Figure 1. The Model of Hollow Solenoid Coils

\subsection{Circuit Model of WPT System}

The circuit model of WPT system is envisaged in Figure 2. The peak value of AC voltage source is $310 \mathrm{~V}$ and the frequency is $50 \mathrm{~Hz}$; the reactance of the primary and secondary circuit are respectively $Z_{1}, Z_{2}$, while the current are respectively $I_{1}$ and $I_{2}$; the load is $R_{L}$; the axial distance is $\mathrm{d}$ and the mutual inductance is $M$.

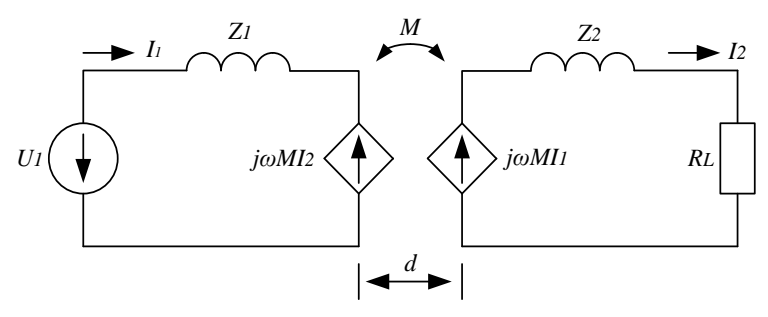

Figure 2. Circuit Model of WPT System

KVL equation is obtained according to the system model

$$
\left\{\begin{array}{c}
I_{1} Z_{1}-j \omega M I_{2}=U_{1} \\
I_{2} Z_{2}-j \omega M I_{1}=0
\end{array}\right.
$$




$$
\begin{gathered}
P_{\text {in }}=\frac{U_{1}^{2} Z_{2}}{Z_{1} Z_{2}+(\omega M)^{2}} \\
P_{\text {out }}=\frac{U_{1}^{2}(\omega M)^{2} R_{L}}{\left[Z_{1} Z_{2}+(\omega M)^{2}\right]^{2}} \\
\eta=(\omega M)^{2} R_{L} /\left(R_{2}+R_{L}\right)\left[R_{1}\left(R_{2}+R_{L}\right)+(\omega M)^{2}\right] \\
\eta=\omega^{2} R_{L} /\left(R_{1}+R_{2}\right)\left[\omega^{2}+\frac{R_{1}\left(R_{2}+R_{L}\right)}{M^{2}}\right]
\end{gathered}
$$

It is obvious in equation (2) that the system efficiency is only related to the mutual inductance when load, excitation source and coils parameters are determined.

2.1.1. Open-circuit Voltage Method: Through 2D FEA of ANSYS, reference [15] gives the analytical method and open-circuit method to calculate mutual inductance to calculate mutual inductance, and compares the two methods. The results illustrates that the relative error of the mutual inductance by the two methods is extremely small. Moreover, the method of open-circuit voltage is more convenient for establishing model and analyzing process.

The paper also takes the method of open-circuit voltage to calculate mutual inductance, however, through 3D FEA of MAGNET.

When the secondary circuit is open

$$
M=U_{2}^{\prime} / \omega I_{1}^{\prime}
$$

Where

$I_{1}^{\prime}$ - the primary peak current when the secondary circuit is open

$U_{2}^{\prime}$ - the secondary peak voltage when the secondary circuit is open

$\omega$-angular frequency

$Z_{1}$ - the reactance of the primary circuit

$Z_{2}$ - the reactance of the secondary circuit

$M$-mutual inductance

\subsection{Sinusoidal Steady-state Simulation Analysis of the Coupling Coils with Open Circuit for the Secondary}

Transmission distance is a crucial index in the WPT system based on the electromagnetic coupling mode. The paper establish 3D model by SOLIDWORKS, and takes the method based on 3D field and circuit coupling to analyze the ration between mutual inductance and axial distance by MAGNET. The model of the coaxial coils and 3D model established by SOLIDWORKS are shown in Figure 3.

$$
R_{1}=R_{3}, R_{2}=R_{4}
$$



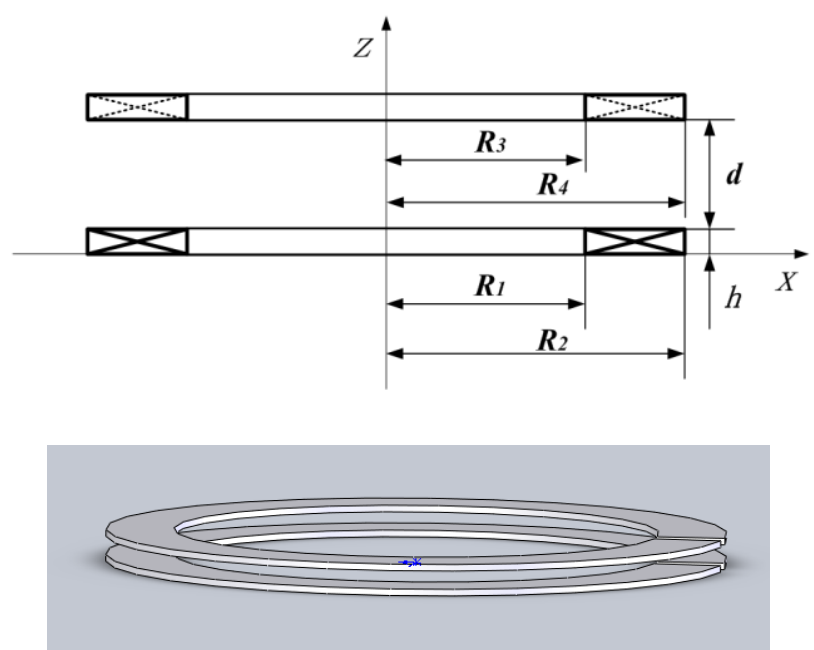

Figure 3. The Model of Coaxial Coupling Coils

2.2.1. The Relation between Mutual Inductance and Axial Distance of Coils: The paper takes open-circuit voltage method to calculate mutual inductance. Take $\mathrm{d}=10 \mathrm{~cm}$ for example, the distribution of magnetic flux is shown in Figure 4.

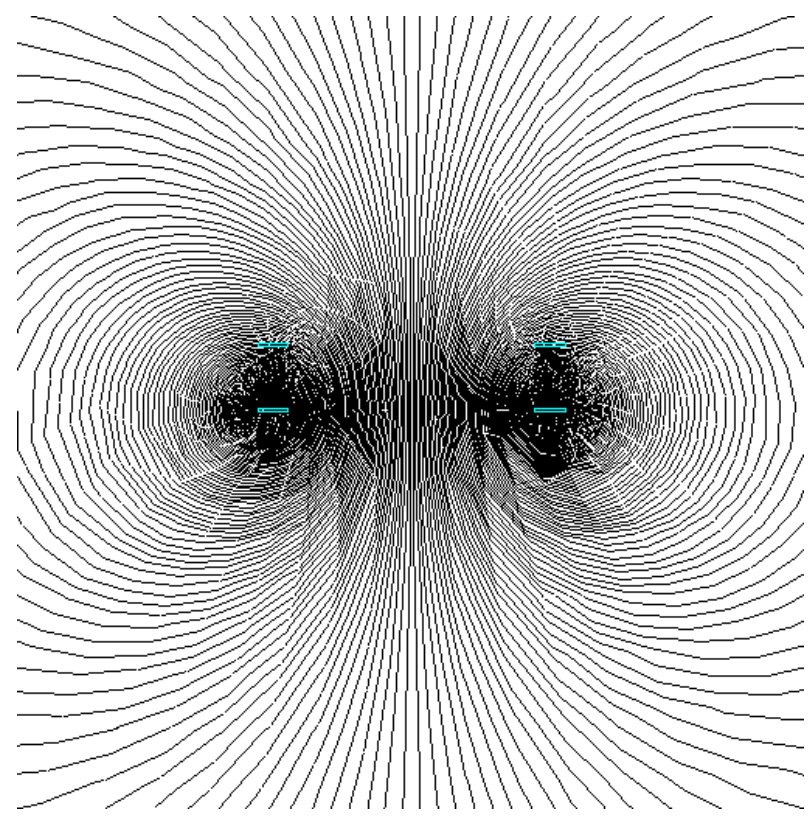

Figure 4. The Distribution of Magnetic Flux when the Secondary Circuit is Open

Figure 5 presents the curve that $M$ varies with $d$. It's easy to see that mutual inductance decreases rapidly when axial distance is small, however, the mutual inductance tends to be steady after $d=8 \mathrm{~cm}$. Equation (4) presents a formula by calculating based on MATLAB and the comparison between the results of simulation and calculation is shown in Figure 5. 


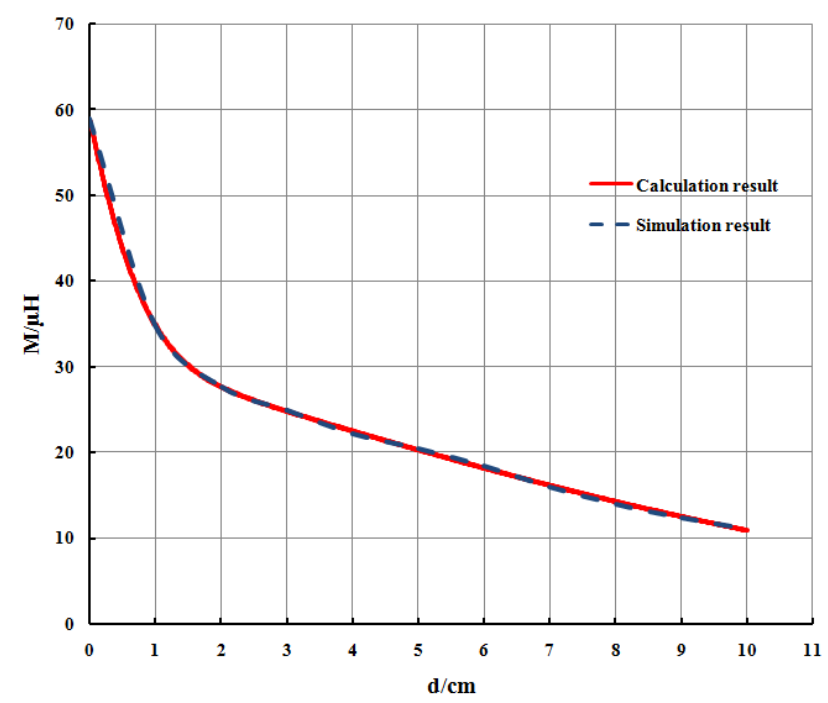

Figure 5. The Comparison of Simulation and Calculation Results

$$
M=f(d)=9 e^{-\left(\frac{d+2}{2}\right)^{2}}+5 e^{-\left(\frac{d+10}{17}\right)^{2}}
$$

\section{Multivariate Analysis for Mutual Inductance of Coupling Coils}

The paper also takes open-circuit voltage method to analyze the variety law of mutual inductance under other conditions and the results can be directly used as the basis for calculating the operation parameters and optimizing the system efficiency

\subsection{The Relation between Mutual Inductance and Cross Section Area of the Secondary Coil}

The model of coupling coils and 3D SOLIDWORKS are shown in Figure 6. The paper analyzes the variety law of mutual inductance under the condition that cross section area of the secondary coil varies while the cross-sectional area of the primary coil doesn't $(d=10 \mathrm{~cm})$. Figure 7 illustrates the curve that p.u. of $\mathrm{M}$ varies with p.u. of the secondary coil's cross-section area. Obviously, the mutual inductance doesn't have maximum value when $\mathrm{S}_{\mathrm{p}}=\mathrm{S}_{\mathrm{s}}$, however, when $S_{s}=1.4 S_{p}$ the mutual inductance has maximum value. The mutual inductance sharply increases on the condition of $S_{s}<1.4 S_{p}$ and decreases when $S_{s}>1.4 S_{p}$. Equation (5) presents a formula by calculating based on MATLAB and the comparison between the results of simulation and calculation is shown in Figure 7.

$\mathbf{S}_{\mathbf{p}}$ - the cross section area of the primary coil

$\mathbf{S}_{\mathbf{s}}$ - the cross section area of the secondary coil 

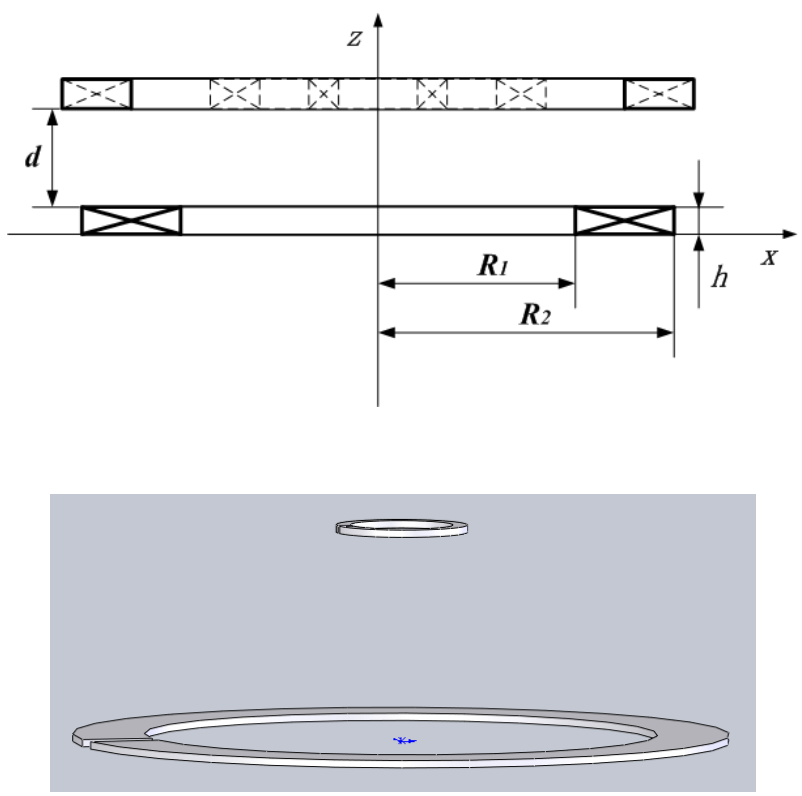

Figure 6. The Coaxial Coupling Coils Model with Different Cross Section Areas of the Secondary Coil

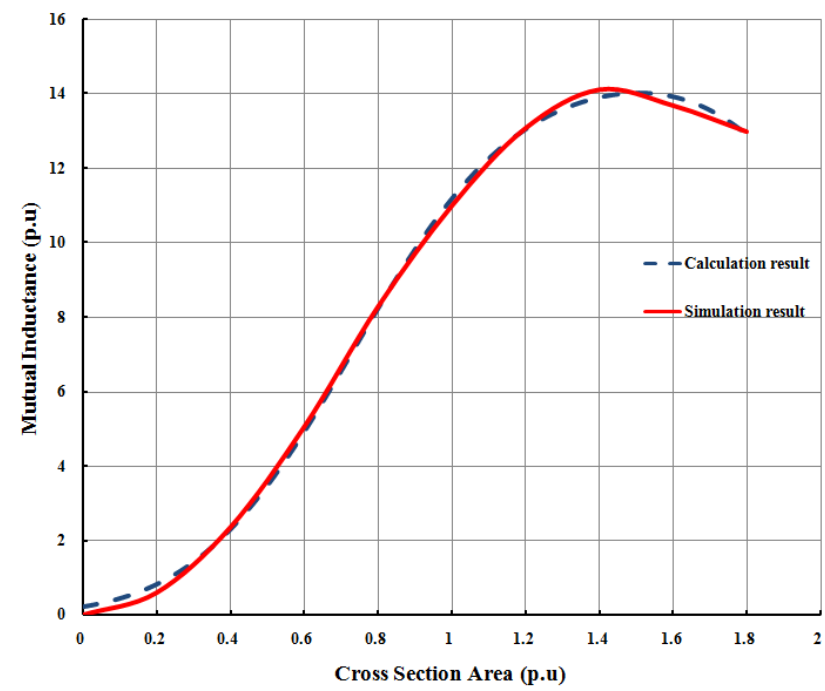

Figure 7. The Comparison of Simulation and Calculation Results

$$
M_{p u}=f\left(S_{p u}\right)=6 e^{-4\left(s_{p u}-1\right)^{2}}+13 e^{-\left(s_{p u}-2\right)^{2}}
$$

The embedded model of coupling coils is shown in Figure 8. The paper analyzes the variety law of mutual inductance. The comparison of simulation and calculation results is shown in Figure 9. It is visible that $M_{p u}$ increases slightly when $S_{p u}<0.4$, and sharply when $0.4<S_{p u}<1$. When $S_{p u}>1, M_{p u}$ decreases rapidly. It is easy to see that $M_{p u}$ has maximum value when $S_{p u}=1$. Equation (6) presents a formula calculating based on MATLAB and the comparison between the results of simulation and calculation is shown in Figure 9. 

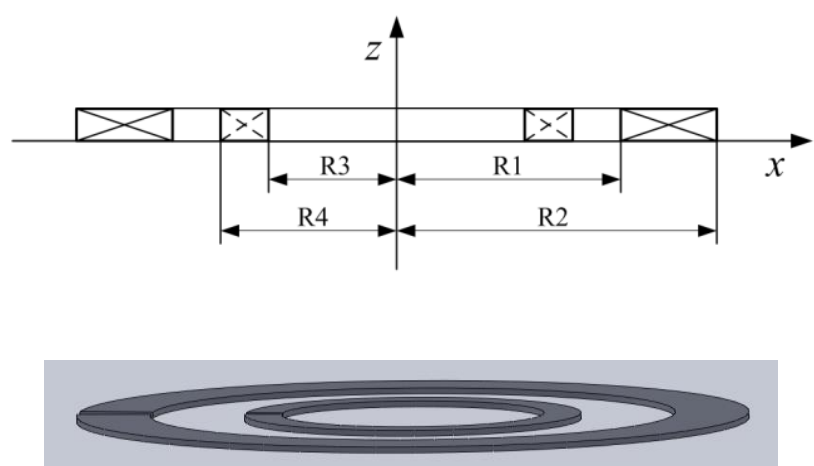

Figure 8. The Embedded Model of Coupling Coils

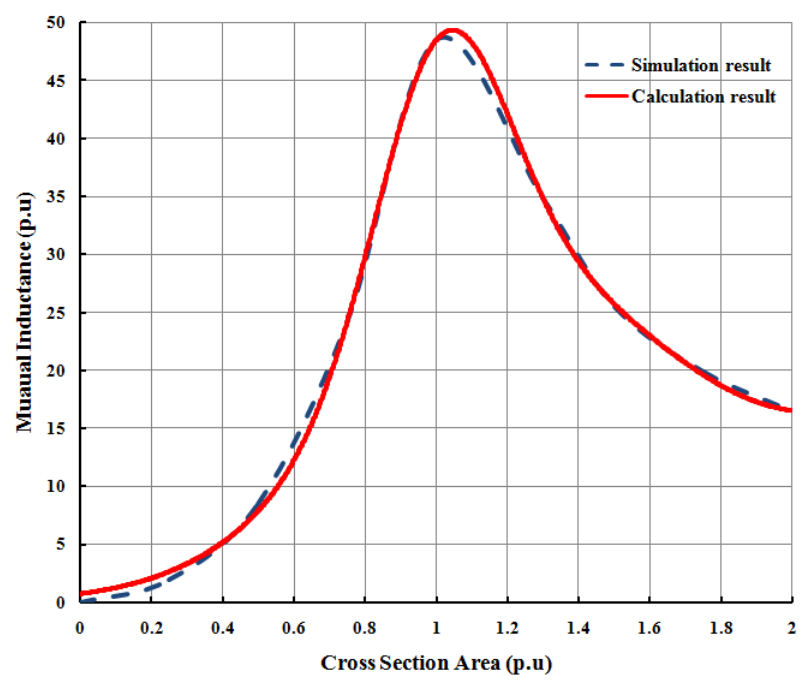

Figure 9. The Comparison of Simulation and Calculation Results

$M_{p u}=f\left(S_{p u}\right)=28.08 e^{\left[-\left(\frac{S_{p u}-3.352}{1.447}\right)^{2}\right]}+24.52 e^{\left[-\left(\frac{S_{p u}-1.204}{0.6224}\right)^{2}\right]}+24.51 e^{\left[-\left(\frac{S_{p u}-1.013}{0.2584}\right)^{2}\right]}$

(6)

\subsection{The Relation between Mutual Inductance and Angular Deviation}

The coupling coils model with included angle is shown in Figure 10. The paper analyzes the variety law of mutual inductance under the condition that angle deviation between coupling coils changes from $0^{\circ}$ to $90^{\circ}$ and the axial distance of the coils is $5 \mathrm{~cm}$. Figure 11 illustrates the curve that $M$ varies with $\alpha$. It is obvious that the mutual inductance decreases sharply when $0^{\circ}<\alpha<15^{\circ}$. The mutual inductance decreases slightly on the condition of $15^{\circ}<\alpha<45^{\circ}$. when $45^{\circ}<\alpha<75^{\circ}$, the mutual inductance decreases rapidly again, however, increases slowly when $75^{\circ}<\alpha<90^{\circ}$. Equation (7) presents a formula by calculating based on MATLAB and the comparison between the results of simulation and calculation is shown in Figure 11. 

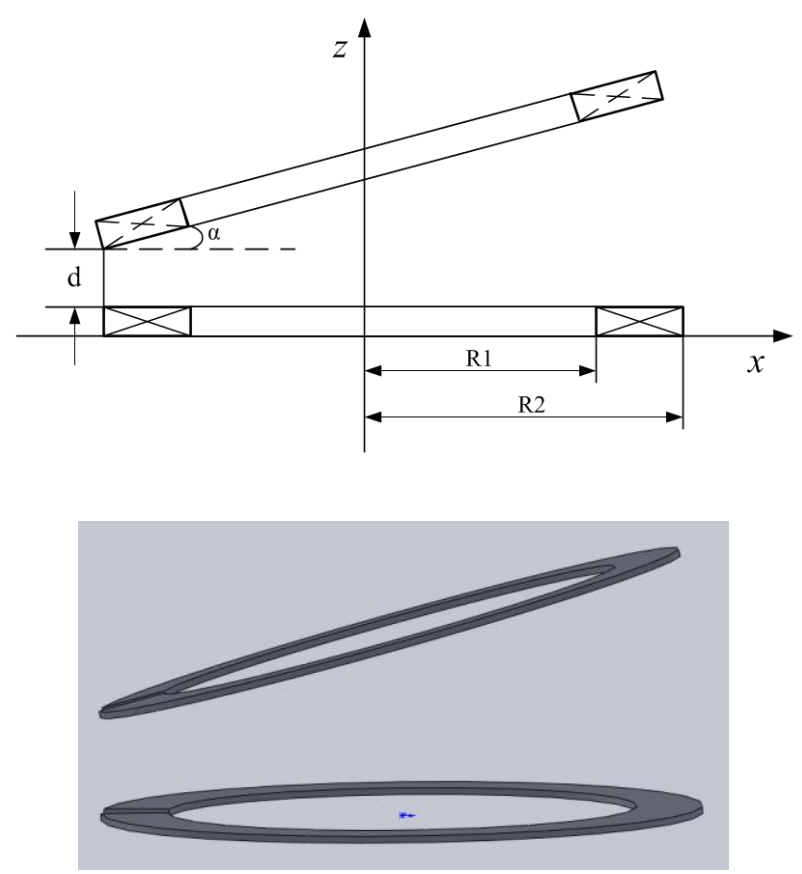

Figure 10. The Model of Coupling Coils having Included Angle

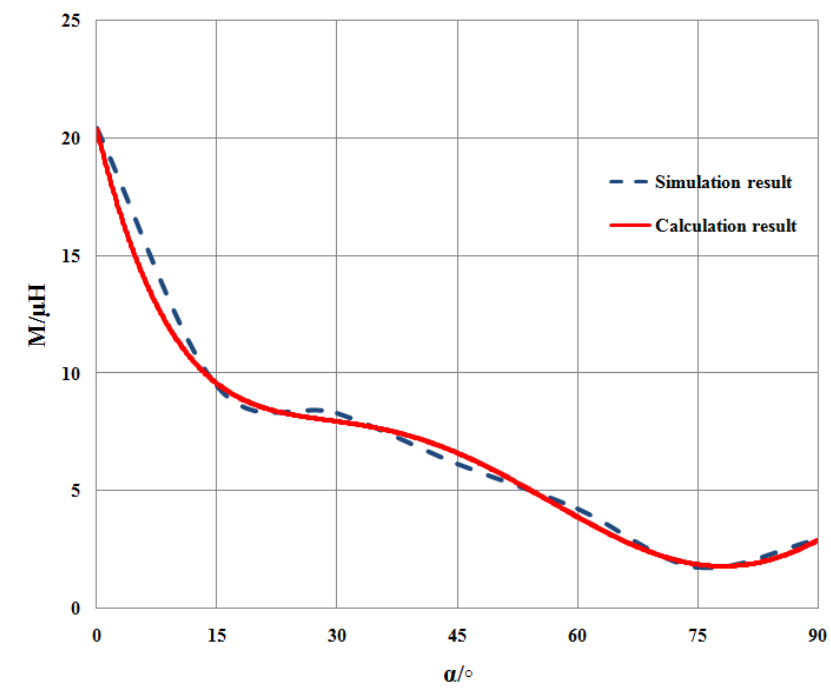

Figure 11. The Comparison of Simulation and Calculation Results

$$
M=f(\alpha)=2 e^{9}-2 e^{9} \cos \alpha+4 e^{7} \sin \alpha+7 e^{8} \cos (2 \alpha)-2 e^{7} \sin (2 \alpha)
$$

\subsection{The Relation between Mutual Inductance and Lateral Deviation}

The model of coupling coils and 3D SOLIDWORKS are shown in Figure 12. The paper analyzes the variety law of mutual inductance with the condition that the lateral deviation changes from $0 \mathrm{~cm}$ to $32 \mathrm{~cm}$. Figure 13 illustrates the curve that $\mathrm{M}$ varies with $\mathrm{s}$. It is obvious that the mutual inductance decrease rapidly when $s<22 \mathrm{~cm}$, however, when $s>22 \mathrm{~cm}$, mutual inductance fluctuates within a narrow range. Equation (8) presents a formula by calculating based on 
MATLAB and the comparison between the results of simulation and calculation is shown in Figure 13.
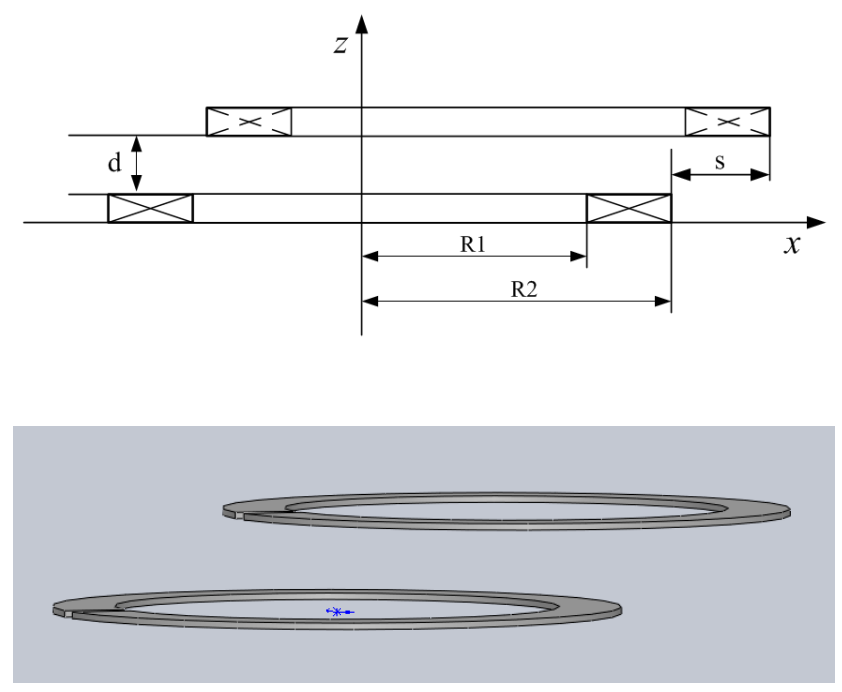

Figure 12. The Model of Coupling Coils with Lateral Deviation

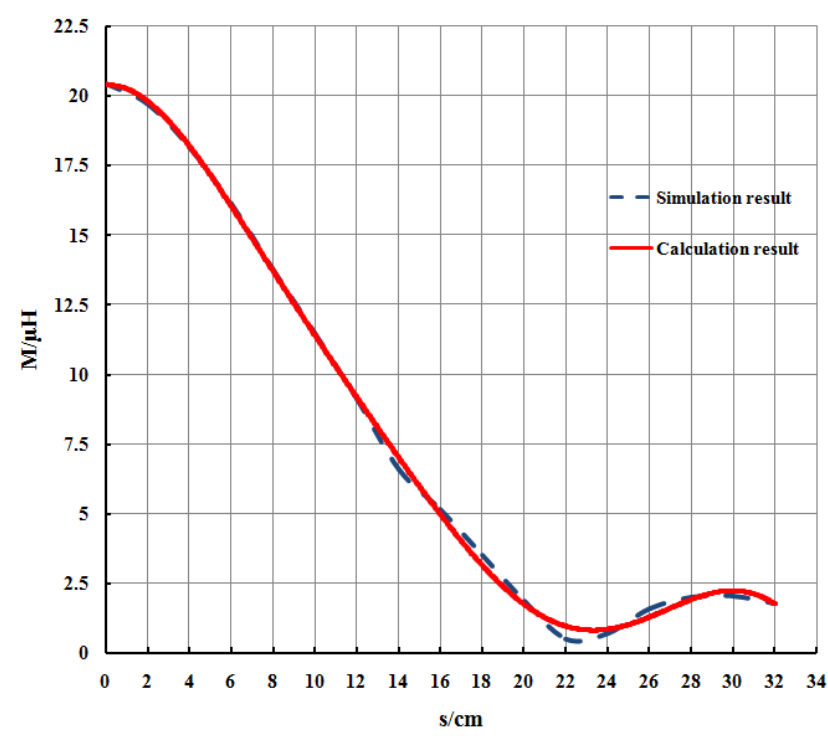

Figure 13. The Comparison of Simulation and Calculation Results

$$
\begin{aligned}
M=f(x)=5 & +9 \cos (0.1 s) \\
& +6 \sin (0.1 s) \\
& +7 \cos (0.2 s)+0.1 \sin (0.2 s)-0.1 \cos (0.3 s) 2 \sin (0.3 s)
\end{aligned}
$$

\section{Conclusion}

The paper illustrates that mutual inductance is a crucial factor in the WPT system based on the electromagnetic coupling mode and then proposes a novel method based on 3D field and circuit coupling analysis. The new method aims at calculating the mutual inductance with different coils parameters, arbitrary spatial distribution. According to the results, mutual inductance can be increased by decreasing the axial distance and the lateral deviation, equaling the cross-section areas of the primary and secondary coil, 
moreover, it is helpful for obtaining maximum efficiency under the condition that the spatial distribution of coupling coils are irregular and restrained in actual physical systems. The paper doesn't make the analysis under the condition that the hollow solenoid coil with magnetic materials, however, the method can be imitated to calculate the mutual inductance of coupling coils. Compared with other approaches, this method is more direct and universal. The method can be directly used for WPT system design and efficiency optimization based on the understanding of the relations among mutual inductance and the variables described above.

\section{References}

[1] S. Hasanzadeh and S. V. Zadeh, "Design of a wireless power transfer system for high power moving applications", Electromagnetics Research M, vol. 28, (2013), pp. 258-271.

[2] H. Qiang, W. Y. Zhu and J. F. Zheng, "Simulation Study on Coil Design of Wireless Power Transfer System for Optimal Transmission Efficiency", Research Journal of Applied Sciences, Engineering and Technology, vol. 5, no. 21, (2013), pp. 5039-5041.

[3] L. L. Tan, X. L. Huang, H. Huang, Y. W. Zou and H. Li, 'Transfer efficiency optimal control of magnetic resonance coupled system of wireless power transfer based on frequency control", Science China Technological Sciences, vol. 54, no. 6, (2013), pp. 1428-1434.

[4] X. L. Huang, H. Qiang and L. L. Tan, "The Coil Misalignment Model of Inductively Coupled Wireless Power Transfer System: Mutual Inductance Analysis and Transfer Efficiency Optimization”, Progress In Electromagnetics Research Symposium Proceedings, (2012); Moscow, Russia.

[5] K. A. Grajski, R. Tseng and C. Wheatley, "Loosely-Coupled Wireless Power Transfer: Physics, Circuits, Standards, Technologies, Systems and Applications (IMWS)”, IEEE MTT-S International, (2012).

[6] Y. Lu, K. Wai, E. Cheng, S. X. Wang, K. Ding and K. W. Kwok, "Design of a contactless power charger for a functional jacket", International Journal of Circuit Theory and Applications, (2011).

[7] S. Valtchev, B. Borges, K. Brandisky and J. B. Klaassens, "Resonant Contactless Energy TransferWith Improved Efficiency", IEEE Transactions on Power Electronics, vol. 24, no. 3, (2009).

[8] H. Rakouth, J. Absmeier, A. Brown Jr., I. S. Suh, J. M. Miller, R. Sumner and R. Henderson, "EV Charging Through Wireless Power Transfer: Analysis of Efficiency Optimization and Technology Trends", SAE-China and FISITA (eds.), Proceedings of the FISITA World Automotive Congress, Lecture Notes in Electrical Engineering, (2012).

[9] I. Awai and T. Ishida, "Design of Resonator-Coupled Wireless Power Transfer System by Use of BPF Theory", Journal of the Korean Institute of Electromagnetic Engineering and Science, vol. 10, no. 4, (2010).

[10] X. H. Li, H. R. Zhang, F. Peng, Y. Li, T. Y. Yang, B. Wang and D. M. Fang, "A Wireless Magnetic Resonance Energy Transfer System for Micro Implantable Medical Sensors", Sensors, vo. 12, (2012).

[11] S. I. Babic and C. Akyel, "New Mutual Inductance Calculation of the Magnetically Coupled Coils: Thin Disk Coil-Thin Wall Solenoid”, J. of Electromagn. Waves and Appl., vol. 20, no. 10, (2006), pp. 1281-1290.

[12] Y. Ren, F. T. Wang, G. L. Kuang, W. G. Chen, Y. F. Tan, J. W. Zhu and P. He, "Mutual Inductance and Force Calculations Between Coaxial Bitter Coils and Superconducting Coils with Rectangular Cross Section”, J Supercond Nov. Magn., vol. 24, (2011), pp. 1687-1691.

[13] T. Župan, Ž. Štih and B. Trkulja, "Fast and Robust Method for Mutual Inductance Calculation of Coaxial Circular Coils with Rectangular Cross Section", Compumag, (2013).

[14] K. Yamaguchi, I. Hodaka and Y. Yamamoto, "Estimation of coupling coefficient for wireless power transfer", Recent Advances in Circuits, Communications and Signal Processing, (2013).

[15] S.Y. Han and X. M. Wang, "Calculation of Mutual Inductance Based on Field-Circuit Coupling Analysis for WPT”, International Conference on Power Electronics Systems and Applications, (2013). 